\title{
Time of day accounts for overnight improvement in sequence learning
}

\author{
Aysha Keisler, ${ }^{1,4}$ James Ashe, ${ }^{2,3}$ and Daniel T. Willingham ${ }^{1}$ \\ ${ }^{1}$ Department of Psychology, University of Virginia, Charlottesville, Virginia 22904, USA; ${ }^{2}$ Brain Sciences Center (11B), \\ Veterans Affairs Medical Center, Minneapolis, Minnesota 55417, USA; ${ }^{3}$ Department of Neuroscience, University of Minnesota, \\ Minneapolis, Minnesota 55455, USA
}

\begin{abstract}
The theory that certain skills improve with a night of sleep has received considerable interest in recent years. However, because sleep typically occurs at the same time of day in humans, it is difficult to separate the effects of sleep from those of time of day. By using a version of the Serial Response Time Task, we assessed the role of sleep in implicit sequence learning while controlling for possible time-of-day effects. We replicated the apparent benefit of sleep on human participants. However, our data show that sleep does not affect implicit sequence learning; rather, time of day affects the ability of participants to express what they have learned.
\end{abstract}

Although we usually associate learning with practice, skill learning is best considered a multiphase process in that improvement may occur even after training ends (Karni and Sagi 1993; Karni et al. 1998; Press et al. 2005). Recent observations of improved performance on a variety of tasks after a night of sleep led to the theory that sleep plays a special role in this process, namely, that memory improves with sleep (Karni et al. 1994; Stickgold et al. 2000; Fischer et al. 2002; Walker et al. 2003; Robertson et al. 2004; Drosopoulos et al. 2005). The role of sleep in memory formation is the topic of considerable controversy, however. Researchers in this field undertake the formidable task of demonstrating that improvements after sleep are not due to circadian influences, reduction in fatigue, the simple passage of time, and a host of other potential confounds. For this and other reasons, some researchers are reticent to accept the theory that sleep improves memory (Siegel 2001; Vertes 2004; Vertes and Siegel 2005).

One of the most daunting methodological dilemmas inherent in this field involves circadian rhythms. Because circadian rhythms dictate when most of us sleep, sleep and time of day are naturally confounded. Typically, researchers either manipulate sleep itself (Karni et al. 1994; Stickgold et al. 2000), manipulate the time of learning relative to sleep (Walker et al. 2003; Huber et al. 2004; Robertson et al. 2004), or some combination of both (Fischer et al. 2002; Drosopoulos et al. 2005; Gais et al. 2006) to measure learning before and after sleep. In other words, researchers either deprive participants of sleep or allow participants to sleep normally and measure learning at particular times of the day. A drawback of the former method is that we cannot discriminate between adverse effects of sleep deprivation (and associated stress) on learning and a beneficial effect of sleep on learning. In other words, even after controlling for fatigue effects at test (i.e., Stickgold et al. 2000), we cannot be sure whether sleep improves memories or a period of sleep deprivation impairs recently acquired memories. A drawback of the latter method is that it fails to control for the possibility of circadian, or time of day, effects, which are known to affect a variety of skills (Cajochen et al. 2004; Hasher et al. 2005).

The fact that that sleep is confounded with circadian cycle is not a new observation, and researchers have applied various ingenious techniques to address this issue. One approach is to train

\section{${ }^{4}$ Corresponding author.}

E-mail aysha@virginia.edu; fax (434) 982-4766.

Article is online at http://www.learnmem.org/cgi/doi/10.1101//m.751807. participants at different times of day but test them at the same time of day (Fenn et al. 2003; Walker et al. 2003; Gais et al. 2006). Another approach is to allow some participants to nap after daytime training while others remain awake (Mednick et al. 2003; Gais et al. 2006; Tucker et al. 2006). These methods, while informative, are not without shortcomings. By training participants at the same time but testing at different times, one confounds sleep with test delay (i.e., the delay between training and test). Naps are informative when an effect of sleep is observed, but a null result is difficult to interpret: Is it that sleep plays no role in learning or that a full night's sleep is necessary to gain the benefit? Furthermore, participants in these studies may differ in degree of fatigue after training. In summary, it is extremely difficult to control for these various factors simultaneously, and control of one variable typically comes at the expense of another. Nevertheless, converging evidence, drawn from various methodologies, suggests that sleep plays a role in at least some types of skill acquisition.

The current research investigates the role of sleep in implicit sequence learning, or sequence learning that takes place outside of awareness (Graf and Schacter 1985). In this task, participants respond to a series of stimuli that, unbeknownst to them, follow a repeating pattern (Nissen and Bullemer 1987). Evidence concerning the role of sleep in implicit sequence learning is mixed. Although some data indicate that sleep facilitates learning (Maquet, et al. 2000; Peigneux et al. 2003), other find no evidence of sleep-related improvement (Robertson et al. 2004; Fischer et al. 2006). We sought to measure the effect of sleep on sequence learning while circumventing the typical difficulties associated with sleep research. We devised a new method to control for possible time-of-day effects, while at the same time allowing participants to sleep normally during the experiment.

We observed learning at many different times on two consecutive days. We trained three groups of participants for eight sessions, with each group beginning at a different time of day, but with all participants experiencing normal sleep and an overnight delay. In this design, a given session occurred at a different time of day for each group, and the groups differed in terms of whether or not sleep occurred before a given session. Thus, while we still could not simultaneously control for sleep and circadian factors, we could consider each factor separately. Specifically, this design allowed us to (1) evaluate potential time-of-day effects independently of the amount of practice, and (2) measure the effect of sleep independently of the amount of practice. 
Forty-five participants with no known sleep disorders volunteered for the study and received $\$ 50.00$ compensation (20 males, $M_{\text {age }}=27.8 \mathrm{yr}, S D_{\text {age }}=3.1 \mathrm{yr}$ ). We asked each participant to choose two consecutive days on which he or she could sleep normally the night before for the study days. Participants slept an average of $7.73 \mathrm{~h}(S D=1.76)$ the night before day 1 and 7.55 $\mathrm{h}(S D=1.28)$ the night before day 2 . Subjective sleep quality was reported on a 1-7 scale, where 1 is "I didn't sleep at all," and 7 is "I had a perfect night of sleep." Mean subjective sleep quality was $5.68(S D=1.20)$ the night before day 1 and $5.17(S D=0.95)$ the night before day 2. Also, we administered the Pittsburgh Sleep Quality Index (Buysse et al. 1989) to assess general sleep quality. Participants' mean score was $4.7(S D=2.3)$ out of 21 possible points, comparable to the mean score of college students without sleep difficulties $(M=4.8, S D=2.2)$ (Yang et al. 2003).

We administered the Alternating Serial Response Time Task (Fig. 1; Howard and Howard 1997), a variation of the Serial Response Time Task (Nissen and Bullemer 1987). A row of four $3-\mathrm{cm} \times 3-\mathrm{cm}$ boxes appeared on a computer screen, and participants were shown four corresponding response keys on the keyboard. A circle appeared in one box until the participant pressed the corresponding response key. The participant's response triggered the presentation of the next stimulus after an interval of $120 \mathrm{msec}$. Participants were instructed to respond as quickly as possible. Unbeknownst to participants, every-other stimulus appeared in a repeating four-element sequence. The remaining stimulus locations were random.

Fifteen participants were randomly assigned to each of the three groups. The Early, Mid, and Late groups, respectively, began practice at 12:00 p.m., 4:00 p.m., and 8:00 p.m. on day 1 . All participants practiced every $2 \mathrm{~h}$ until 10:00 p.m. On day 2 , the groups continued to train every $2 \mathrm{~h}$, beginning at 9:00 a.m., until each had completed eight sessions in all. Thus, participants in each group underwent identical amounts of training but because they began training at different times, sleep occurred at different points in the learning curve. Furthermore, because participants at a particular time of day had undergone different amounts of practice, we could dissociate time of day and degree of training. (This design differs from previous sleep studies in that we observe skill acquisition over many sessions rather than compare performance at two points [i.e., before and after sleep or a comparable delay]. However, there is no reason to suppose a qualitative dif-

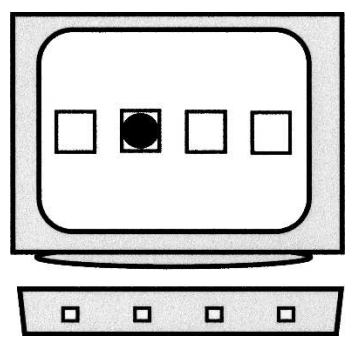

Figure 1. Schematic of Alternating Serial Response Time Task. The response keys were the $\mathrm{s}, \mathrm{f}, \mathrm{h}$, and $\mathrm{k}$ buttons and mapped to the boxes so that the leftmost response key corresponded to the leftmost box, and so on. Participants were instructed to respond as quickly and accurately as possible to the location of a stimulus by pressing the corresponding key on a keyboard. The stimulus appeared according to a repeating secondorder sequence. For example, take the repeating sequence $2-1-3-4 \ldots$. . in which 1 represents a stimulus presentation in the left-most box, 4 represents the right-most box, etc. The participant would see the series $2 r 1 r 3 r 4 r 2 r 1 r 3 r 4 r$, where $r$ represents one of the four positions chosen at random. A sequence was randomly assigned to each participant from a list of 22 possible four-unit sequences that met the following constraints: each stimulus location (1-4) appeared once and only once, and there were no ascending or descending runs (i.e., 4-3-2-1). Post-experiment interviews showed that no participant became aware of the sequence.
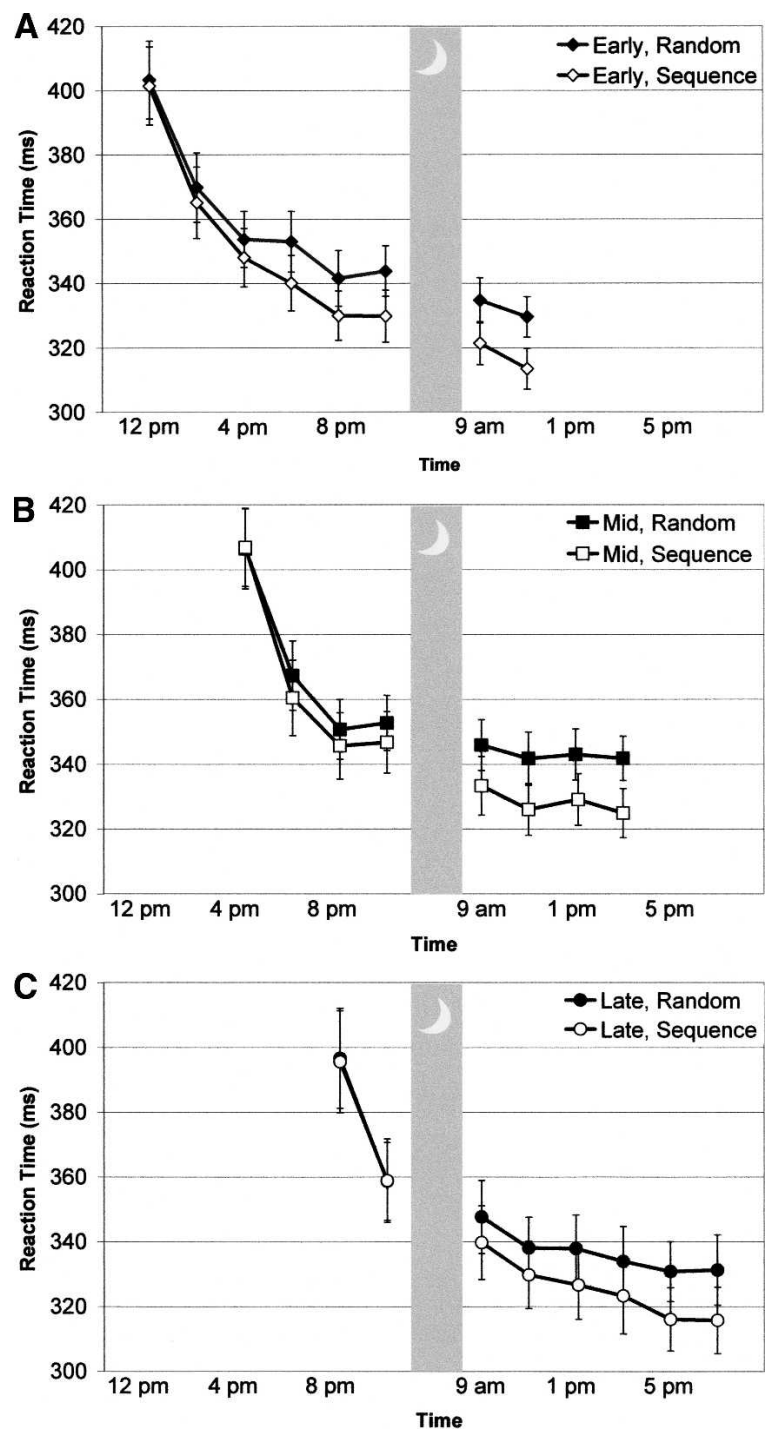

Figure 2. Mean RTs during the Alternating Serial Response Time Task across time. Each session consisted of 500 trials, grouped in ten 50-trial blocks. The first 10 trials of each block were entirely random, and the remaining 40 trials consisted of alternating sequenced and random stimuli (five sequence presentations). Thus, in each session each participant responded to 300 random trials and 200 sequenced trials.

ference in skill acquisition between these two scenarios, or more importantly, a difference in the effect of sleep on memory.)

Figure 2, A-C, shows participants' reaction times (RTs) across the eight practice sessions. Figure 3A shows participants' learning scores, or RT differences between random and sequence trials, across the eight practice sessions. First, we compared improvement between sessions that contained sleep to improvement between sessions that did not contain sleep. The Mid group improves between day 1 and day 2, $t=2.47, P<0.05$, but the Early and Late groups do not improve between the corresponding sessions (i.e., sessions 4 and 5), $P>0.20$. Similarly, the Late group improves between day 1 and day $2, t=3.19, P<0.05$, but the Early and Mid groups do not improve between the corresponding sessions (i.e., sessions 2 and 3 ), $P>0.20$. This difference is the typical phenomenon highlighted in sleep studies. The Early group does not improve overnight, likely because their performance approached asymptote.

To further assess the effect of sleep, we analyzed learning 

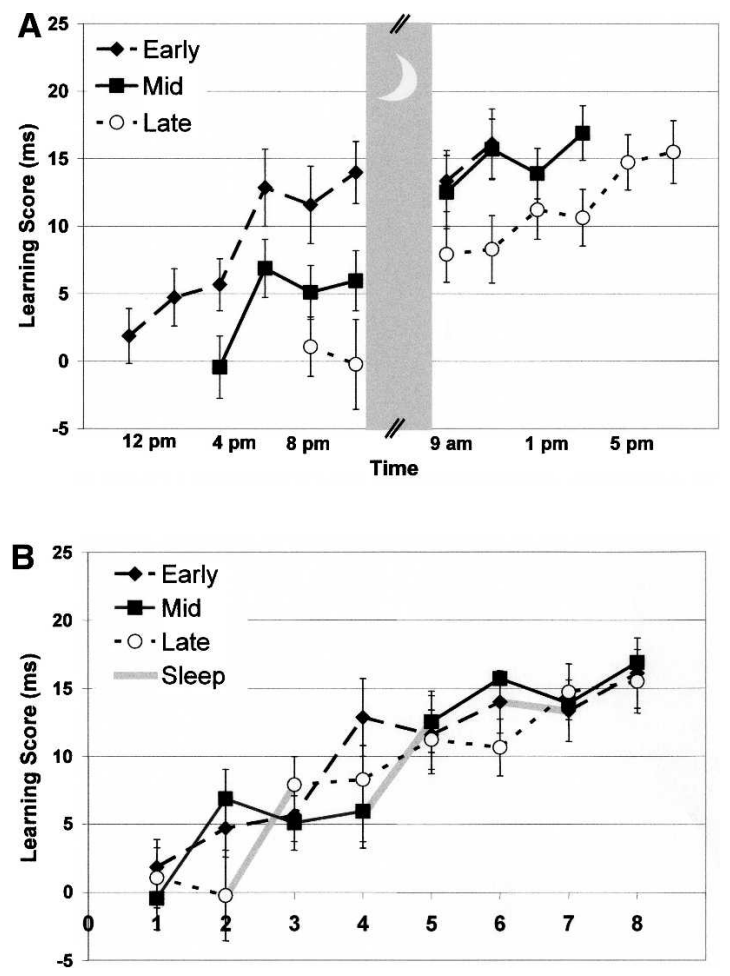

Figure 3. Learning scores during the Alternating Serial Response Time Task. We calculated the median response time of each presentation of the four-unit sequence and each presentation of the four alternating random trials. Thus, there were 50 sequence medians and 50 random medians per session per participant. We then calculated the mean of each set of medians and subtracted the sequence mean from random mean. This procedure yielded a single learning score for each session for every participant. $(A)$ Learning scores across time. The gray bar indicates the overnight break, during which participants slept at home. Note that no group shows improvement in learning after 6:00 PM. $(P>0.2)$. (B) Learning scores across session. These are the same data shown in $A$, reformatted to enable comparison by sessions. Gray bars between data points denote the overnight break between sessions.

scores across sessions to determine whether there was a discontinuity associated with the overnight break, as would be expected if sleep benefits learning. Analysis of learning scores including session and day as independent nested variables revealed a significant effect of session for each group $(F s=22.9,24.1$, and 19.6 for the Early, Mid, and Late groups, respectively, $P$ s $<0.01$ ), indicating that learning increased with training. Furthermore, there was a significant effect of day in the Mid and Late groups ( $F \mathrm{~s}=16.0$ and 19.8, respectively, $P \mathrm{~s}<0.01$ ), indicating that learning increased overnight in addition to across-session improvement. Although the Early group showed a significant effect of session $(F=22.9, P<0.01)$, this group did not show an effect of day $(F=0.20, P>0.20)$, indicating that they did not show overnight improvement. Again, this group likely approached asymptotic performance for the task on day 1 . Thus, we replicated the overnight benefit on performance in two of our three groups.

If the overnight effect were due to sleep, participants who had slept should perform better than those that had not at the same point in training (i.e., participants that had completed the same number of sessions). For instance, the Late group should have performed better than the other groups during session 3 , as only participants in the Late group had slept by this session (see Fig. 3B). Similarly, the Early group should have performed worse than the other groups during session 5, as only participants in the Early group had not yet slept by this session. Neither prediction is confirmed in one-way ANOVAs ( $\left.P^{\prime} \mathrm{s}>0.20\right)$. Thus, while participants improved overnight, this improvement did not translate to a performance benefit compared to participants with the same amount of practice. How can sleep apparently yield substantial overnight improvement within a group and yet no advantage compared to groups that have not slept?

The explanation for this apparent paradox is clear if one notes that no group improved after 6:00 PM on day $1(P \mathrm{~s}>0.20)$. Indeed, although groups differed in absolute learning scores, learning curves were strikingly flat and parallel at 6:00 p.m., 8:00 p.m., and 10:00 p.m. (Fig. 3A). However, performance in the morning was equivalent to other groups during the same session (Fig. 3B). Therefore, our data suggest that learning occurs during the evening sessions but is not expressed in performance. In other words, participants continue to learn in the evening hours even though their performance does not improve.

In the current experiment, performance on a sequencing task was depressed in the evening. However, in the morning, the negative time-of-day influence was no longer a factor, and performance returned to that which would be expected, given the degree of practice. If performance is only measured immediately before and after sleep, as in previous studies, the overnight change could be mistakenly attributed to sleep. In fact, our data demonstrate that learning does not improve with a night of sleep.

Although this interpretation may seem peculiar at first, it must be remembered that performance is not a transparent window into learning but combines learning and other factors such as motivation, fatigue, attention, etc. (Matthews et al. 2000). Multiple studies illustrate the dissociation between expression of learning and learning itself. For instance, a long response-tostimulus interval impairs expression of sequence learning but not learning (Willingham et al. 1997). Similar discussions surrounded the roles of secondary tasks (Frensch et al. 1998, 1999; Shanks and Channon 2002) and attention (Jiang and Leung 2005) in learning versus expression. Furthermore, different brain structures may support learning and its expression (Seidler et al. 2002). A requirement of learning and memory studies is that performance must be compared between identical test conditions (Willingham et al. 1997). The current results highlight that systematic variance in time of day introduces a significant confound.

An alternative explanation of the current results is that participants did not actually gain sequence knowledge in the evening, but they did improve overnight. In other words, rather than an effect of time of day on expression of learning, perhaps there was an effect of time of day on learning as well as a subsequent effect of sleep on learning. In this scenario, however, the magnitude of the evening impairment would have to be identical, coincidentally, to the overnight learning gain for both the Mid group and the Early group. A far more likely interpretation is that learning occurs in the evening but expression of that learning is impeded by time of day.

Why is performance impaired at night? While many tasks show time-of-day effects (Carrier and Monk 2000; Hasher et al. 2005), the cause of these fluctuations is poorly understood. At least two factors contribute to time-of-day effects: "sleep debt," or the length of time spent awake, and endogenous circadian effects, or the time at which behavior is observed. Separating the contributions of these two factors is difficult and typically involves complex manipulations of participants' environment and schedule (Carrier and Monk 2000; Cajochen et al. 2004).

We demonstrate that, due to inherent methodological difficulties in sleep research, circadian influences can be misattributed to a sleep effect. There is no reason to assume that circadian 
or sleep influences are homogenous across all skills, of course, and our data should not be taken to mean that sleep does not improve any skills. However, the current results do call previous evidence of the sleep effect into question: To the extent that circadian influences were not adequately controlled, we cannot be sure that sleep accounts for behavioral differences before and after sleep. Future studies should evaluate the relative contributions of sleep and time of day in other areas of sleep research, such as visual discrimination learning (i.e., Stickgold et al. 2000) and declarative learning (i.e., Drosopoulos et al. 2005).

Importantly, the fact that certain memories do not improve with sleep does not mean that sleep and cognition are unrelated. It has been shown that sleep properties are affected by learning earlier in the day (Skaggs and McNaughton 1996; Huber et al. 2004; Ribeiro and Nicolelis 2004) and that sleep characteristics can be correlated with performance (Walker et al. 2002; Huber et al. 2004). The current research focused on memory improvement, but sleep may change other properties of memory and learning. Also, perhaps these results are better interpreted to indicate that learning influences certain characteristics of sleep, but sleep does not influence learning. Clearly, there is much left to unravel concerning sleep, memory, and circadian effects, and multiple approaches are necessary to elucidate these complex interactions.

\section{References}

Buysse, D.J., Reynolds III, C.F., Monk, T.H., Berman, S.R., and Kupfer, D.J. 1989. The Pittsburgh Sleep Quality Index: A new instrument for psychiatric practice and research. Psychiatry Res. 28: 193-213.

Cajochen, C., Knoblauch, V., Wirz-Justice, A., Kräuchi, K., Graw, P., and Wallach, D. 2004. Circadian modulation of sequence learning under high and low sleep pressure conditions. Behav. Brain Res. 151: $167-176$.

Carrier, J. and Monk, T.H. 2000. Circadian rhythms of performance: New trends. Chronobiol. Int. 17: 719-732.

Drosopoulos, S., Wagner, U., and Born, J. 2005. Sleep enhances explicit recollection in recognition memory. Learn. Mem. 12: 44-51.

Fenn, K.M., Nusbaum, H.C., and Margoliash, D. 2003. Consolidation during sleep of perceptual learning of spoken language. Nature 425: $614-616$

Fischer, S., Hallschmid, M., Elsner, A.L., and Born, J. 2002. Sleep forms memory for finger skills. Proc. Natl. Acad. Sci. 99: 11987-11991.

Fischer, S., Drosopoulos, S., Tsen, J., and Born, J. 2006. Implicit learning-explicit knowing: A role for sleep in memory system interaction. J. Cogn. Neurosci. 18: 311-319.

Frensch, P.A., Lin, J., and Buchner, A. 1998. Learning versus behavioural expression of the learned: The effects of a secondary tone-counting task on implicit learning in the serial reaction task. Psychol. Res. 61: 83-98.

Frensch, P.A., Wenke, D., and Rünger, D. 1999. A secondary tone-counting task suppresses expression of knowledge in the serial reaction task. J. Exp. Psychol. Learn. Mem. Cogn. 12: 260-274.

Gais, S., Lucas, B., and Born, J. 2006. Sleep after learning aids memory recall. Learn. Mem. 13: 259-262.

Graf, P. and Schacter, D.L. 1985. Implicit and explicit memory for new associations in normal and amnesic subjects. J. Exp. Psychol. Learn. Mem. Cogn. 11: 501-518.

Hasher, L., Goldstein, D., and May, C. 2005. It's about time: Circadian rhythms, memory, and aging. In Human learning and memory: Advances in theory and application (eds. C. Izawa and N. Ohta), pp. 199-217. Lawrence Erlbaum Associates, Mahwah, NJ.

Howard Jr., J.H. and Howard, D.V. 1997. Age differences in implicit learning of higher order dependencies in serial patterns. Psychol. Aging 12: 634-656.

Huber, R., Ghilardi, M.F., Massimini, M., and Tononi, G. 2004. Local sleep and learning. Nature 430: 78-81.

Jiang, Y. and Leung, A.W. 2005. Implicit learning of ignored visual context. Psychon. Bull. Rev. 12: 100-106.

Karni, A. and Sagi, D. 1993. The time course of learning a visual skill. Nature 365: 250-252.

Karni, A., Tanne, D., Rubenstein, B.S., Askenasy, J.J.M., and Sagi, D. 1994. Dependence on REM sleep of overnight improvement of a perceptual skill. Science 265: 679-682.

Karni, A., Meyer, G., Rey-Hipolito, C., Jezzard, P., Adams, M.M., Turner, R., and Ungerleider, L.G. 1998. The acquisition of skilled motor performance: Fast and slow experience-driven changes in primary motor cortex. Proc. Natl. Acad. Sci. 95: 861-868.

Maquet, P., Laureys, S., Peigneux, P., Fuchs, S., Petiau, C., Phillips, C., Aerts, J., Del Fiore, G., Degueldre, C., Meulemans, T., et al. 2000. Experience-dependent changes in cerebral activation during human REM sleep. Nat. Neurosci. 3: 831-836.

Matthews, G., Davies, R.D., Westerman, S.J., and Stammers, R.B. 2000. Human performance: Cognition, stress and individual differences. Taylor and Francis, Philadelphia, PA.

Mednick, S., Nakayama, K., and Stickgold, R. 2003. Sleep-dependent learning: A nap is as good as a night. Nat. Neurosci. 6: 697-698.

Nissen, M.J. and Bullemer, P. 1987. Attention requirements of learning: Evidence from performance measures. Cognit. Psychol. 19: 1-32.

Peigneux, P., Laureys, S., Fuchs, S., Destrebecqz, A., Collette, F., Delbeuck, X., Phillips, C., Aerts, J., Del Fiore, G., Degueldre, C., et al. 2003. Learned material content and acquisition level modulate cerebral reactivation during posttraining rapid-eye-movements sleep. Neuroimage 20: 125-134.

Press, D.Z., Casement, M.D., Pascual-Leone, A., and Robertson, E.M. 2005. The time course of off-line motor sequence learning. Brain Res. Cogn. Brain Res. 25: 375-378.

Ribeiro, S. and Nicolelis, M.A.L. 2004. Reverberation, storage, and postsynaptic propagation of memories during sleep. Learn. Mem. 11: $686-696$.

Robertson, E.M., Pascual-Leone, A., and Press, D.Z. 2004. Awareness modifies the skill-learning benefits of sleep. Curr. Biol. 114: 208-212.

Seidler, R.D., Purushotham, A., Kim, S.G., Uğurbil, K., Willingham, D., and Ashe, J. 2002. Cerebellum activation associated with performance change but not motor learning. Science 296: 2043-2046.

Shanks, D.R. and Channon, S. 2002. Effects of a secondary task on "implicit" sequence learning: Learning or performance. Psychol. Res. 66: 99-109.

Siegel, J.M. 2001. The REM sleep-memory consolidation hypothesis. Science 294: 1058-1063.

Skaggs, W.E. and McNaughton, B.L. 1996. Replay of neuronal firing sequences in rat hippocampus during sleep following spatial experience. Science 271: 1870-1873.

Stickgold, R., James, L., and Hobson, J.A. 2000. Visual discrimination learning requires sleep after training. Nat. Neurosci. 3: 11237-11238.

Tucker, M.A., Hirota, Y., Wamsley, E.J., Lau, H., Chaklader, A., and Fishbein, W. 2006. A daytime nap containing solely non-REM sleep enhances declarative but not procedural memory. Neurobiol. Learn. Mem. 86: 241-247.

Vertes, R.P. 2004. Memory consolidation in sleep; dream or reality. Neuron 44: $135-148$.

Vertes, R.P. and Siegel, J.M. 2005. Time for the sleep community to take a critical look at the purported role of sleep in memory processing. Sleep 28: 1228-1229.

Walker, M.P., Brakefield, T., Morgan, A., Hobson, J.A., and Stickgold, R. 2002. Practice with sleep makes perfect: Sleep-dependent motor learning. Neuron 35: 205-211.

Walker, M.P., Brakefield, T., Hobson, J.A., and Stickgold, R. 2003. Dissociable stages of human memory consolidation and reconsolidation. Nature 425: 616-620.

Willingham, D.B., Greenberg, A.R., and Thomas, R.C. 1997. Response-to-stimulus interval does not affect implicit motor sequence learning, but does affect performance. Mem. Cognit. 25: 534-542.

Yang, C., Wu, C., Hsieh, M., Liu, M., and Lu, F. 2003. Coping with sleep disturbances among young adults: A survey of first-year college students in Taiwan. Behav. Med. 29: 133-138.

Received August 23, 2007; accepted in revised form August 31, 2007. 


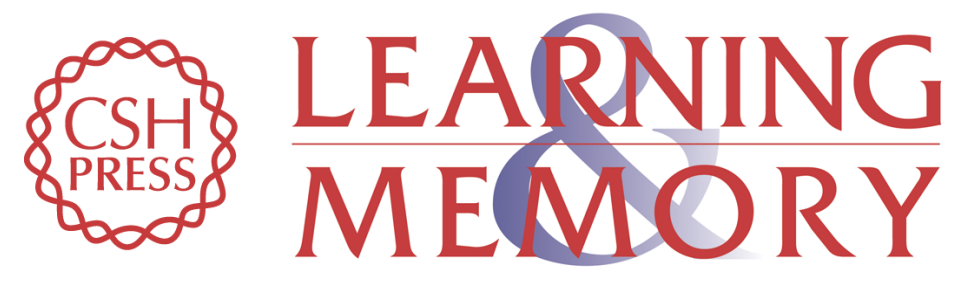

\section{Time of day accounts for overnight improvement in sequence learning}

Aysha Keisler, James Ashe and Daniel T. Willingham

Learn. Mem. 2007, 14:

Access the most recent version at doi:10.1101/lm.751807

References This article cites 36 articles, 9 of which can be accessed free at:

http://learnmem.cshlp.org/content/14/10/669.full.html\#ref-list-1

License

Email Alerting Receive free email alerts when new articles cite this article - sign up in the box at the Service top right corner of the article or click here. 